\title{
Mental Health Assessment of Youth with Sickle Cell Disease and Their Primary Caregivers During the COVID-19 Pandemic
}

\author{
Nancy Green ${ }^{1}$, Deepa Manwani ${ }^{2}$, Kim Smith-Whitley ${ }^{3}$, Banu Aygun ${ }^{4}$, Abena \\ Appiah-Kubi ${ }^{4}$, and Arlene Smaldone ${ }^{5}$ \\ ${ }^{1}$ Columbia University \\ ${ }^{2}$ Children's Hospital at Montefiore \\ ${ }^{3}$ The Children's Hospital of Philadelphia \\ ${ }^{4}$ Steven and Alexandra Cohen Children's Medical Center \\ ${ }^{5}$ Columbia University Irving Medical Center
}

January 4, 2022

\begin{abstract}
Youth with sickle cell disease (SCD) and their caregivers are susceptible to stress and depression, perhaps exacerbated by pandemic-associated health and economic concerns. Most of the 50 youth-caregiver dyads enrolled in the multi-site HABIT trial took an on-line survey of self-reported mental health symptoms and food insecurity during the 2020 COVID-19 pandemic. Compared to largely pre-pandemic results, prevalence of mental health symptoms in dyad members appeared to have shifted: fewer youth and more caregivers were affected during the pandemic; many of both groups lacked optimism. Pandemic screening of youth with SCD for mental health symptoms and food insecurity appears warranted.
\end{abstract}

Brief Report:

Mental Health Assessment of Youth with Sickle Cell Disease and Their Primary Caregivers During the COVID-19 Pandemic

Nancy S. Green, MD, ${ }^{1,6}$ Deepa Manwani, MD, ${ }^{2}$ Kim Smith-Whitley, MD ${ }^{3}$ Banu Aygun, MD, ${ }^{4}$ Abena Appiah-Kubi, MD, MS ${ }^{4}$ Arlene M. Smaldone, PhD, CPNP-PC, FAAN ${ }^{5}$

1 Division of Hematology, Oncology and Stem cell transplantation, Dept. of Pediatrics, Columbia University Irving Medical Center, NY

2 Division of Hematology and Oncology, Dept. of Pediatrics, Albert Einstein College of Medicine, NY

3 Division of Hematology and Oncology and Stem cell transplantation, Children's Hospital of Philadelphia, PA

4 Division of Hematology and Oncology and Cellular Therapy, Cohen Children's Medical Center, New Hyde Park, NY

5 School of Nursing and College of Dental Medicine, Columbia University Irving Medical Center, NY

6 Corresponding author:

650 West 168 St., Box 168

New York, NY 10032 
nsg11@cumc.columbia.edu

212-305-0494

Word count: 100 Text: 1200 words Tables: 2

Short running title: Pandemic Mental Health in Youth and Caretakers

Key words: Sickle cell disease, pandemic, youth, mental health, depression, anxiety

Abbreviations key:

\begin{tabular}{ll}
\hline SCD & Sickle cell disease \\
\hline U.S. & United States \\
HABIT trial & $\begin{array}{l}\text { Hydroxyurea Adherence for Personal Best in Sickle Cell Treatment } \\
\text { SVI }\end{array}$ \\
\hline
\end{tabular}

Note: This work was previously presented as "Mental Health Assessment of Youth with Sickle Cell Disease and Their Primary Caretakers: Baseline Depression and COVID-19 Pandemic-Associated Psychosocial Stress in a Multi-Site Study" at the December 2020 meeting of the American Society of Hematology, Blood (2020) 136 (Supplement 1): 41-42 (doi.org/10.1182/blood-2020-140866).

\section{Acknowledgements :}

This work was funded by 1R01NR017206 (Green, Smaldone)

We acknowledge the expertise of Andrew Rundles, DrPH and James W. Quinn at the Columbia Mailman School of Public Health for calculating the Social Vulnerability Index of this sample. We thank the patients with SCD and their caregivers who participated in the HABIT Trial and these surveys, as well as the study staff at each participating study site.

Conflict of Interest Statement : The authors have no relevant conflicts to disclose.

\section{Abstract}

Youth with sickle cell disease (SCD) and their caregivers are susceptible to stress and depression, perhaps exacerbated by pandemic-associated health and economic concerns. Most of the 50 youth-caregiver dyads enrolled in the multi-site HABIT trial took an on-line survey of self-reported mental health symptoms and food insecurity during the 2020 COVID-19 pandemic. Compared to largely pre-pandemic results, prevalence of mental health symptoms in dyad members appeared to have shifted: fewer youth and more caregivers were affected during the pandemic; many of both groups lacked optimism. Pandemic screening of youth with SCD for mental health symptoms and food insecurity appears warranted.

\section{Introduction}

Most people living with sickle cell disease (SCD) in the United States (U.S.) identify as Black and/or Latino, ${ }^{1}$ whose communities are disproportionately under-resourced and vulnerable to adverse social factors. ${ }^{2-4}$ Social, environmental and disease-related factors render youth with SCD and their caregivers susceptible to mental health issues, e.g. stress and depression..$^{5-8}$

Societal disruptions during the 2020 COVID-19 pandemic included prolonged school and workplace closures, social isolation and economic insecurity disproportionately affected communities with lower socio-economic status. ${ }^{9-12}$ People with SCD experienced increased complications and hospitalizations from COVID-19 infection compared to other Blacks. ${ }^{13,14}$ Pandemic-related health concerns may have added to mental health risk among SCD-affected families.

Fifty dyads of youth with SCD ages 10-18 years and their primary caregivers enrolled in our multi-site randomized, controlled trial, HABIT ("Hydroxyurea Adherence for Personal Best in Sickle Cell Treatment"), to 
improve youth's diminished adherence to hydroxyurea therapy. ${ }^{15}$ The HABIT trial was conducted from 2018 through 2021 at four pediatric SCD centers in New York City and Philadelphia. Trial enrollment occurred between May 2018 and December 2020, with 89\% enrolled prior to the March 2020 onset of the COVID-19 pandemic. Participants completed the pediatric and adult PROMIS ${ }^{\circledR}$ measure for depressive symptoms at trial enrollment. ${ }^{16}$ At pandemic onset, study visits were largely converted to virtual platforms. The Social Vulnerability Index (SVI), a composite scale estimating vulnerability to social adversity by U.S. census tract, was calculated using pre-pandemic standards. SVI ranges from 0-1.0; higher values represent greater vulnerability. ${ }^{17}$ Nationally, high SVI, including minority status, was associated with increased COVID-19 infections. ${ }^{18}$

We hypothesized that mental health symptoms were common among HABIT dyads during the early months of the COVID-19 pandemic, and that food insecurity, a measure of economic instability, was frequently experienced. ${ }^{3}$ Enrolled dyads were offered participation in an open-access pandemic-focused online survey of standardized adult core mental health symptoms from June-October, $2020 .{ }^{19}$ We minimally adapted the survey questions for study youth. The pandemic-focused survey obtained self-reported mental health symptoms and behaviors experienced during the preceding week: nervous, anxious or "on edge"; depressed; lonely, or any $>1$ of these; not feeling hopeful about the future; physical reactions (e.g. sweating, nausea, palpitations, or "trouble breathing") when thinking about the pandemic. Reponses of $>1$ days were scored as having symptoms. Questions also gauged changes in school or work arrangements, history of mental health conditions, current substance abuse and experience of verbal abuse. We added a pair of validated screening questions about food insecurity. ${ }^{20}$ Spanish survey translations were available as preferred. Participants identifying specific "red flag" issues (frequent mental health symptoms, food insecurity or substance abuse) were referred to their site's social services. Dyad responses were compared using McNemar's test. Institutional review board approval was obtained at each study site.

\section{Results}

Of HABIT enrolled trial dyads, $36(72 \%)$ youth and 38 (76\%) caregivers completed the survey. No significant demographic differences were found compared to non-participating dyads. Demographic features are as shown (Table 1). Most (81.6\%) primary caregivers were female. The sample's SVI was $0.87 \pm 0.203$, reflecting high social vulnerability. At trial enrollment among this sub-sample (mean $11.8 \pm 5.4$ months prior to the survey), youth (mean age $14.2 \pm 1.9$ years) reported depressive symptoms more frequently than their caregivers: $58.3 \%$ vs. $15.8 \%, \mathrm{p}=.004$.

Eight $(22.2 \%)$ youth in the pandemic survey reported having experienced one or more of the mental health symptoms asked about, most frequently loneliness (Table 2). In contrast to depressive symptoms at HABIT enrollment, fewer youth than caregivers reported depression $(5.6 \%$ vs. $21.1 \%, \mathrm{p}=0.02)$ or anxiety $(2.8 \%$ vs. $23.7 \%, \mathrm{p}=0.008$ ). Despite these differences, approximately half of both groups lacked optimism about the future ("not feeling hopeful"). More youth than caregivers had experienced changes in work or school routines ( $83.3 \%$ vs. $52.6 \%, \mathrm{p}=0.001)$.

Per caregiver survey, two youth $(5.6 \%)$ and 5 caregivers $(13.2 \%)$ had a history of a mental or behavioral condition. Two youth and four caregivers (10.5\%) reported use of a non-medical substance (alcohol, marijuana and/or non-medical drug), and two from each group had experienced verbal abuse from a parent/partner. Three youth (8.4\%) and five caregivers (13.2\%) reported food insecurity.

In six dyads (17.6\%), one or both members reported one or more "red flag" issues, including daily mental health symptoms, substance or verbal abuse, and/or food insecurity and were referred to their site's social service resources for follow up and intervention if needed.

\section{Discussion:}

Using a self-administered standardized assessment of mental health symptoms during the initial months of COVID-19 pandemic, we assessed youth with SCD and their caregivers from a sample of dyads enrolled in our hydroxyurea adherence trial. We had hypothesized that pandemic-related social and health concerns 
would precipitate depression and other mental health symptoms. Comparing largely pre-pandemic results from a different self-assessment tool of depressive symptoms, prevalence of mental health symptoms appeared to have shifted over time: fewer youth and more caregivers were affected during the pandemic. Nonetheless, a substantial proportion of both groups lacked optimism about the future.

Mental health responses may have reflected pandemic-associated burdens, living with SCD and/or social stressors associated with social vulnerability. In a survey comparing adult childhood cancer survivors to their unaffected siblings, the former more frequently reported health concerns and social isolation. Those data suggest that mental health concerns can be exacerbated by feelings of vulnerability from chronic illness. ${ }^{21}$

Established associations exist between depression and medication non-adherence in chronic illnesses of youth and adults, including adolescents living with SCD. ${ }^{22-24}$ Hence, the finding of frequent depression at trial enrollment was not surprising. Interestingly, fewer youth reported mental health symptoms in the pandemic survey. This result lead us to speculate that some regular social stressors may have lessened during the pandemic, even as stressors affected more caregivers. Nonetheless, youth depression scores in our sample at enrollment were only modestly higher than those reported in a sample with SCD of similar age prescribed hydroxyurea at another pediatric SCD center. ${ }^{25}$ These similarities in findings suggest that depression in adolescents with SCD is common, as recently reported from a large multinational survey of adults with SCD. ${ }^{26}$

Mental health symptoms among U.S. adults during the COVID-19 pandemic significantly increased compared to pre-pandemic levels, with especially high frequencies among those of minoritized racial and ethnic groups. ${ }^{27,28}$ Adolescents overall appear to be sensitive to the pandemic's social impacts, and families affected by SCD may be especially vulnerable. ${ }^{29}$

Study limitations included the modest sample size with limited statistical power to detect differences. Survey participation was offered to the convenient sample of HABIT participants rather than broadly to youth with SCD and their primary caregivers at participating clinical sites. Use of two different self-reported assessment tools pre- and during the pandemic prevented direct comparisons. Survey questions adjusted for youth participants lacked independent validation. Respondents may have slanted responses towards those perceived as more socially acceptable regarding mental health symptoms and/or food insecurity. Our methodology precluded further probing about the mental health symptoms and their potential impact on health, quality of life or other features.

In conclusion, screening for mental health symptoms, social disruption, verbal abuse and food insecurity may be warranted in this high-risk group of social and medical vulnerability, especially during the pandemic. ${ }^{30}$ Interventions for these families, e.g., state or federal aid programs and/or available mental health services, may help support the health and mental health of youth with SCD and their caregivers.

\section{References}

1. Hassell KL. Population estimates of sickle cell disease in the U.S. Am J Prev Med 2010;38:S512-21.

2. Lee L, Smith-Whitley K, Banks S, Puckrein G. Reducing Health Care Disparities in Sickle Cell Disease: A Review. Public Health Rep 2019;134:599-607.

3. Fernandez CR, Licursi M, Wolf R, Lee MT, Green NS. Food insecurity, housing instability, and dietary quality among children with sickle cell disease: Assessment from a single urban center. Pediatr Blood Cancer 2021:e29463.

4. Cronin RM, Hankins JS, Byrd J, et al. Risk factors for hospitalizations and readmissions among individuals with sickle cell disease: results of a U.S. survey study. Hematology 2019;24:189-98.

5. Sil S, Woodward KE, Johnson YL, Dampier C, Cohen LL. Parental Psychosocial Distress in Pediatric Sickle Cell Disease and Chronic Pain. J Pediatr Psychol 2021;46:557-69. 
6. Yarboi J, Compas BE, Brody GH, et al. Association of social-environmental factors with cognitive function in children with sickle cell disease. Child Neuropsychol 2017;23:343-60.

7. Jonassaint CR, Jones VL, Leong S, Frierson GM. A systematic review of the association between depression and health care utilization in children and adults with sickle cell disease. Br J Haematol 2016;174:136-47.

8. Varni JW, Panepinto JA. Cognitive functioning, patient health communication, and worry mediate pain predictive effects on health-related quality of life in youth with sickle cell disease. Pediatr Blood Cancer 2020;67:e28680.

9. Kim B, Rundle AG, Goodwin ATS, et al. COVID-19 testing, case, and death rates and spatial sociodemographics in New York City: An ecological analysis as of June 2020. Health Place 2021;68:102539.

10. Lee CM, Cadigan JM, Rhew IC. Increases in Loneliness Among Young Adults During the COVID-19 Pandemic and Association With Increases in Mental Health Problems. J Adolesc Health 2020;67:714-7.

11. Nagata JM, Ganson KT, Whittle HJ, et al. Food Insufficiency and Mental Health in the U.S. During the COVID-19 Pandemic. Am J Prev Med 2021;60:453-61.

12. Jia H, Guerin RJ, Barile JP, et al. National and State Trends in Anxiety and Depression Severity Scores Among Adults During the COVID-19 Pandemic - United States, 2020-2021. MMWR Morb Mortal Wkly Rep 2021;70:1427-32.

13. Singh A, Brandow AM, Panepinto JA. COVID-19 in individuals with sickle cell disease/trait compared with other Black individuals. Blood Adv 2021;5:1915-21.

14. Panepinto JA, Brandow A, Mucalo L, et al. Coronavirus Disease among Persons with Sickle Cell Disease, United States, March 20-May 21, 2020. Emerg Infect Dis 2020;26:2473-6.

15. Smaldone A, Manwani D, Aygun B, et al. HABIT efficacy and sustainability trial, a multi-center randomized controlled trial to improve hydroxyurea adherence in youth with sickle cell disease: a study protocol. BMC Pediatr 2019;19:354.

16. Dampier C, Barry V, Gross HE, et al. Initial Evaluation of the Pediatric PROMIS(R) Health Domains in Children and Adolescents With Sickle Cell Disease. Pediatr Blood Cancer 2016;63:1031-7.

17. Fletcher KM, Espey J, Grossman MK, et al. Social vulnerability and county stay-at-home behavior during COVID-19 stay-at-home orders, United States, April 7-April 20, 2020. Ann Epidemiol 2021;64:76-82.

18. Karaye IM, Horney JA. The Impact of Social Vulnerability on COVID-19 in the U.S.: An Analysis of Spatially Varying Relationships. Am J Prev Med 2020;59:317-25.

19. McGinty EE, Presskreischer R, Han H, Barry CL. Psychological Distress and Loneliness Reported by US Adults in 2018 and April 2020. JAMA 2020;324:93-4.

20. Hartline-Grafton H, Hassink SG. Food Insecurity and Health: Practices and Policies to Address Food Insecurity among Children. Acad Pediatr 2021;21:205-10.

21. Krull KR, McDonald A, Goodman P, et al. Impact of COVID-19 pandemic on a large cohort of adult survivors of childhood cancer. Pediatr Blood Cancer 2021;68:e29324.

22. Badawy SM, Thompson AA, Penedo FJ, Lai JS, Rychlik K, Liem RI. Barriers to hydroxyurea adherence and health-related quality of life in adolescents and young adults with sickle cell disease. Eur J Haematol 2017;98:608-14.

23. Zheng K, Abraham C, Bruzzese JM, Smaldone A. Longitudinal Relationships Between Depression and Chronic Illness in Adolescents: An Integrative Review. J Pediatr Health Care 2020;34:333-45.

24. DiMatteo MR, Lepper HS, Croghan TW. Depression is a risk factor for noncompliance with medical treatment: meta-analysis of the effects of anxiety and depression on patient adherence. Arch Intern Med 
2000;160:2101-7.

25. Badawy SM, Barrera L, Cai S, Thompson AA. Association between Participants' Characteristics, Patient-Reported Outcomes, and Clinical Outcomes in Youth with Sickle Cell Disease. Biomed Res Int 2018;2018:8296139.

26. Osunkwo I, Andemariam B, Minniti CP, et al. Impact of sickle cell disease on patients' daily lives, symptoms reported, and disease management strategies: Results from the international Sickle Cell World Assessment Survey (SWAY). Am J Hematol 2021;96:404-17.

27. Czeisler ME, Lane RI, Petrosky E, et al. Mental Health, Substance Use, and Suicidal Ideation During the COVID-19 Pandemic - United States, June 24-30, 2020. MMWR Morb Mortal Wkly Rep 2020;69:1049-57.

28. McKnight-Eily LR, Okoro CA, Strine TW, et al. Racial and Ethnic Disparities in the Prevalence of Stress and Worry, Mental Health Conditions, and Increased Substance Use Among Adults During the COVID-19 Pandemic - United States, April and May 2020. MMWR Morb Mortal Wkly Rep 2021;70:162-6.

29. Loades ME, Chatburn E, Higson-Sweeney N, et al. Rapid Systematic Review: The Impact of Social Isolation and Loneliness on the Mental Health of Children and Adolescents in the Context of COVID-19. J Am Acad Child Adolesc Psychiatry 2020;59:1218-39 e3.

30. Power-Hays A, Li S, Mensah A, Sobota A. Universal screening for social determinants of health in pediatric sickle cell disease: A quality-improvement initiative. Pediatr Blood Cancer 2020;67:e28006.

\section{Hosted file}

Green et al. T1 COVID HABIT mental health 1.3.2022.docx available at https://authorea.com/ users/453903/articles/551675-mental-health-assessment-of-youth-with-sickle-cell-diseaseand-their-primary-caregivers-during-the-covid-19-pandemic

\section{Hosted file}

Green et a. T2 COVID HABIT mental health 1.3.2022.docx available at https://authorea.com/ users/453903/articles/551675-mental-health-assessment-of-youth-with-sickle-cell-diseaseand-their-primary-caregivers-during-the-covid-19-pandemic 